\section{Insects and vortices}

\section{R. McNeill Alexander}

The Evolution of Insect Flight. By A. K. Brodsky. Oxford University Press: 1994. Pp. 229. £55, \$82.50.

THE mechanics of insect wing movements can be understood only in the light of the complex anatomy of the thorax and wing bases. The mechanisms of flight present formidable problems in aerodynamics. Happily, Andrei Brodsky is expert in both. He has been doing fine research on insect flight for 25 years but, published in Russian, it was little known in the West

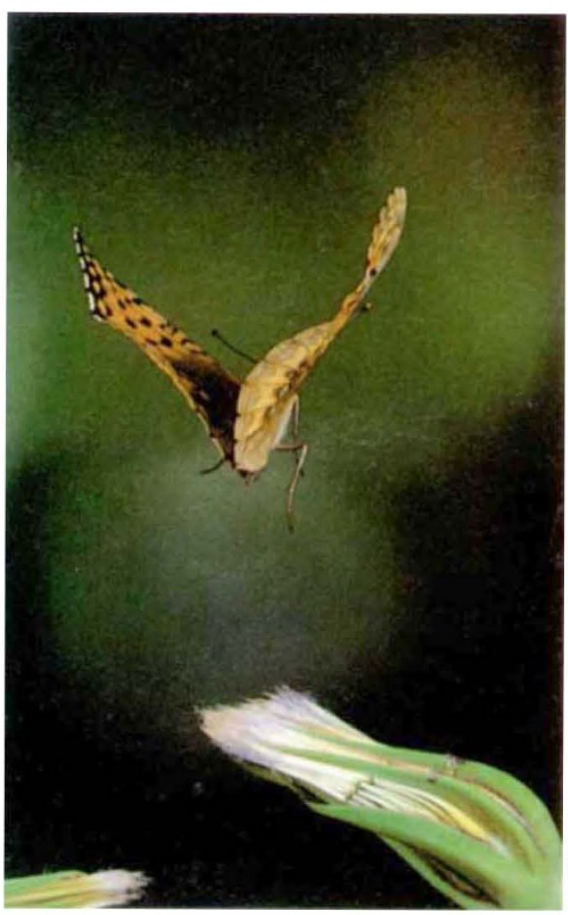

Flying fritillary - taken from John Brackenbury's Insects in Flight (Blandford, 1992; £18.99). His latest book Insects: Life Cycles and the Seasons (Blandford, £19.99), to be published later this month, once again provides a stunning showcase for the author's unique photographic techniques.

until a paper by him appeared in the Journal of Experimental Biology in 1991. There he used spectacular photographs of smoke flowing past the wings of a flying butterfly to reveal the vortices on which flight depends. We have been familiar for several years with the vortex patterns of bird flight; vortex rings produced in the downstroke only at low speeds, and wavy trailing vortices in fast flight. Brodsky has shown that insect flight depends on a different pattern, a chain of linked vortices, one for each up- or downstroke. This results from the circulation of air around the wings being reversed between each stroke and the next, a pattern apparently used by hummingbirds but no other birds.

The book starts with a superb general account of the structure of insect wings and thoraxes, of the mechanism of wing beating and of the kinematics and aerodynamics of flight. It is illustrated by diagrams that time and again gave me a shock of pleasure as I came to understand points much more clearly than I had done before. Other illustrations are complicated drawings that cannot be appreciated without careful study.

The general account of flight occupies a third of the book, and will be widely useful. The rest, on the evolution of flight, will be valuable to specialists who cannot read Brodsky's papers in their original Russian, but most readers will be content to skip the details and extract just the broad picture. Brodsky uses fossil evidence, comparative anatomy and studies of living insects to identify three main lineages of insect flight and nine types of kinematics.

There is no physiology in this book, but for the anatomy, mechanisms and evolution of insect flight it has no rivals.

R. McNeill Alexander is in the Department of Pure and Applied Biology, University of Leeds, Leeds LS2 9JT, UK.

\section{Deep questions, flawed answers}

\section{N. David Mermin}

Observational Foundations of Physics. By Alan Cook. Cambridge University Press: 1994. Pp. 164. £27.95, \$39.95 (hbk); $£ 12.95, \$ 18.95$ (pbk).

THE second is now defined as the duration of $9,192,631,770$ periods of the radiation corresponding to the transition between the two hyperfine levels of the ground state of the caesium-133 atom; since 1991 the metre has been defined as $1 / 299,792,458$ of the distance travelled by light in a second. What implications do these definitions have for the form and content of physical theory?

According to Alan Cook the implications are profound. The first definition requires the time evolution of a quantum mechanical state to be given by a firstorder differential equation, and together they lead to the invariance of the relativistic interval along with the accompanying geometry of four-dimensional vectors. Cook also argues that there is a link between defining time by a classical periodic process and equations of motion of the Hamiltonian form. In all these cases, he suggests, much of the detailed formal structure of theoretical physics may be determined by how we locate things in space and time.

Many other deep questions are examined in this little book. What does chaos in classical dynamical systems do to the notion that the aim of science is to make successful predictions? How should it lead us to reexamine the foundations of statistical mechanics? What, for that matter, is the nature of probability? Is it subjective or objective? Does it deal with frequencies or degrees of belief? Why, when you come to think of it, is mathematics so extraordinarily good at accounting for physical behaviour?

The questions are stimulating and Cook offers some highly original answers. Particularly intriguing (and provocative) is his notion that the remarkable power of mathematics can be traced to the fact that physical systems themselves might be viewed as concrete realizations of the basis vectors for the irreducible representations of groups.

But more often than not I found his answers unpersuasive. The questions are deep and difficult, and a book so short and comprehensive can do little more than point to possible directions. The arguments sketched by Cook, however, often suffer from a lack of clear logical structure - a failure to distinguish between what follows from the premises and what is simply put in by hand.

Furthermore, when an assertion is clear and precise, it is often simply incorrect. Cook states, for example, that a symmetry group describing reflection in a single plane has three members (it has two); he asserts that the number of operations in a space group is finite (it is infinite); he takes as fundamental that it is impossible to observe distant events except with electromagnetic radiation (what about neutrino signals, and why are millions being spent to build gravitational wave detectors?); he declares that a quantum mechanical Hamiltonian must be Hermitian because "all wave functions are orthonormal" (an absurdity - it is because the time evolution must preserve orthonormality); two pages after a statement that the equations of classical dynamics are generally not of a certain form, we are told how they can always be put in just that form. The accumulation of such small infelicities undermines confidence in the broader and more obscure parts of the argument.

This, in short, is a book that takes up many interesting questions but offers answers that are fragmentary and flawed. If it stimulates others to do a better job, it will have served a useful purpose.

N. David Mermin is in the Laboratory of Atomic and Solid State Physics, Cornell University, Clark Hall, Ithaca, New York 14853-2501, USA. 